\title{
Histological differences of skin among three body regions in male and female Hokkaido sika deer (Cervus nippon yesoensis)
}

\author{
By \\ Jun A. MINAGUCHI ${ }^{1)}$, Ayako ABE ${ }^{1)}$, Hiromi UEDA ${ }^{2)}$, Prasarn TANGKAWATTANA ${ }^{3)}$ \\ and Kazushige TAKEHANA ${ }^{1)}$
}

\author{
${ }^{1)}$ Laboratory of Microanatomy, Rakuno Gakuen University, Ebetsu, Hokkaido 069-8501, Japan \\ ${ }^{2)}$ Laboratory of Veterinary Anatomy, Rakuno Gakuen University, Ebetsu, Hokkaido 069-8501, Japan \\ ${ }^{3}$ Department of Veterinary Anatomy, Khon Kaen University, Khon Kaen 40002, Thailand
}

- Received for Publication, February 16, 2012 -

Key Words: Hokkaido sika deer, Skin, Collagen fibrils

\begin{abstract}
Summary: The Hokkaido sika deer (Cervus Nippon yesoensis), the largest and most abundant of the sika deer subspecies in Japan, has recently attracted new attention as a target for leather production, in addition to its meat value. To provide fundamental data for facilitating the effective use of skin for leather, the histological properties of skin at the shoulder, back and abdominal regions of male and female deer were compared. The results showed that the thickness of the outer skin layer was not significantly different across all regions irrespective of sex. Regarding collagen composition, we found that large-diameter collagen fibrils were heavily distributed in the shoulder of male deer, whereas small-diameter collagen fibrils were largely confined to the abdomen of female deer. We hope this regional histological data will lead to more efficient processing of Hokkaido sika deer skin for leather production.
\end{abstract}

\section{Introduction}

In accordance with a change in policy regarding population adjustment of Hokkaido sika deer (Cervus nippon yesoensis), the animal is now classified as a natural resource not only for its meat, but also for using its skin for leather products. For incorporation into Japan Industrial Standards (JIS), we performed experiments concerning leather suitability on the skin of three body regions: shoulder, back, and abdomen. Our study sought to understand the skin's basic structural makeup and characteristics that might give rise to different properties (i.e., strength and flexibility) of the skin, in both male and female deer. As such, differences in collagen fiber components would serve as the fundamental physical basis of these characteristics. Owing to the correlation between morphological features, such as the mean collagen fibril diameter in connective tissues, with macroscopic features, such as the maximum resistance to applied mechanical stress $^{1)}$, the histology of the outer skin layer for each body region of both sexes was explored using both optical microscopy and transmission electron microscopy. The data from our research is beneficial, both for leading to more effective usage of Hokkaido sika deer skin for leather pro- duction, and for generating economic motivation for the population adjustment of Hokkaido sika deer.

\section{Materials and Methods}

In accordance with the Wildlife Protection and Proper Hunting Act, four male and two female Hokkaido sika deer were collected from Nishiokoppe Village, Monbetsu, Hokkaido, from February to April 2010. Skin samples of each deer were taken at the para-median of the right shoulder, back, and abdomen following the prescription detailed in JIS K 6550. After removing the fur and subcutaneous tissue, each sample was fixed using $10 \%$ formalin solution for optical microscopy and using 3\% glutaraldehyde solution with $0.1 \mathrm{M}$ phosphate buffer $(\mathrm{pH}$ 7.4) for electron microscopy. The thickness of the outer skin layer (epidermis and dermis) was measured at nine locations over each paraffinized section using optical microscopy. Further, 600 collagen fibrils from each skin sample were measured using transmission electron microscopy. Image J software (version 1.6, NIH, USA) was used for morphometric analysis. The mass-average diameter (MAD) was calculated to represent the average diameter and toughness ${ }^{2}$. In addition, the number of collagen fibrils 
per unit area was measured from twelve locations in each sample, and the collagen fibril index (CFI) showing the percentage of collagen fibrils was calculated ${ }^{3)}$. The means \pm standard errors of data for the different regions within each sex and between the same regions of different sexes were compared. A Tukey-Kramer multiple comparison test $(\mathrm{P}<0.05)$ was performed.

\section{Results}

Light micrographs of Hokkaido deer skin are shown in Fig. 1. The average thickness in all regions in both sexes was $0.46 \pm 0.01 \mathrm{~mm}$ (Fig. 2). However, the thickness of the outer skin layer for any particular site, regardless of sex, was not significantly different from any other site.
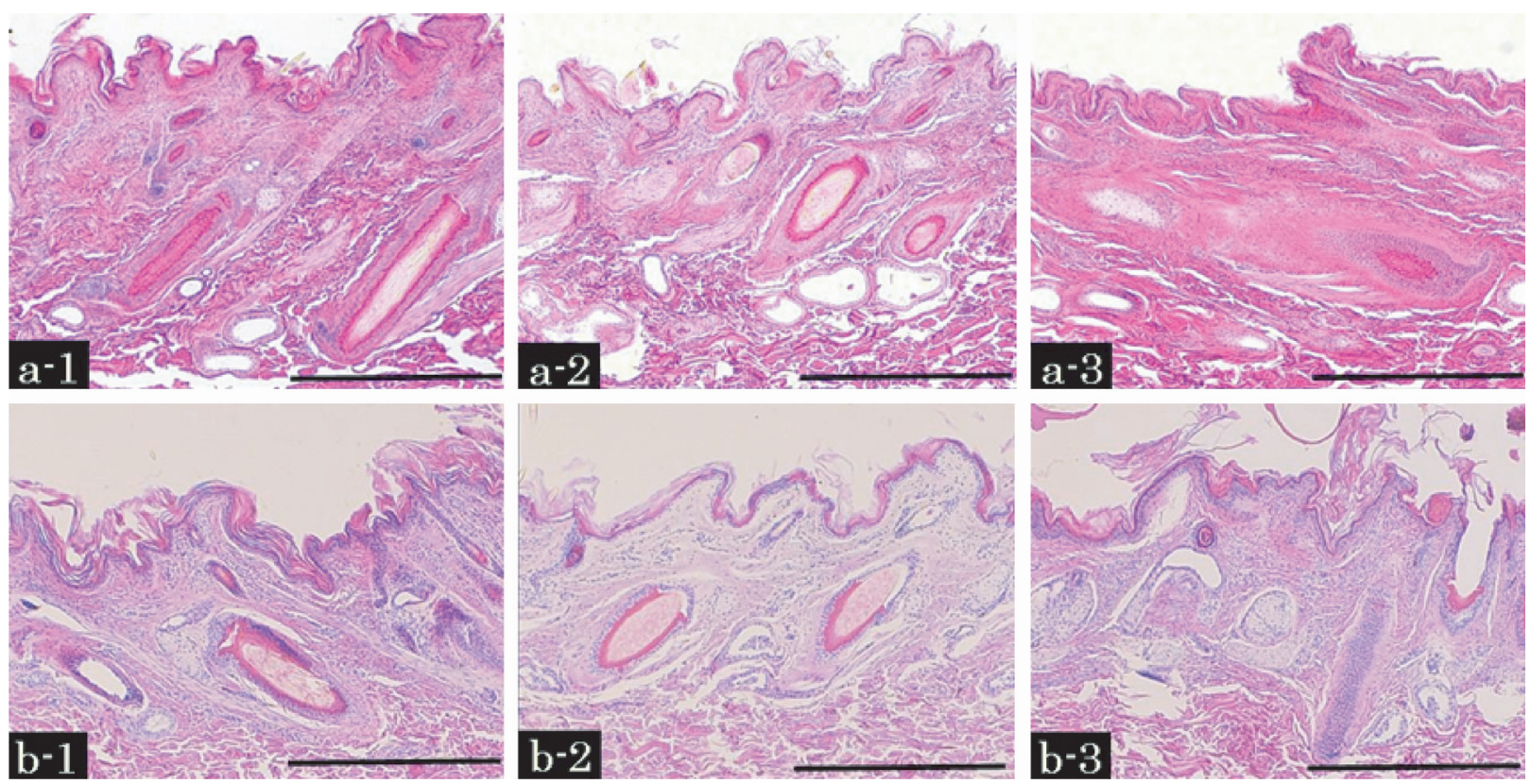

Fig. 1. Light micrographs of Hokkaido sika deer skin. a: Male, b: Female, 1: Shoulder region, 2: Back region, and 3: Abdominal region. Bars indicate a scale of $0.5 \mathrm{~mm}$.

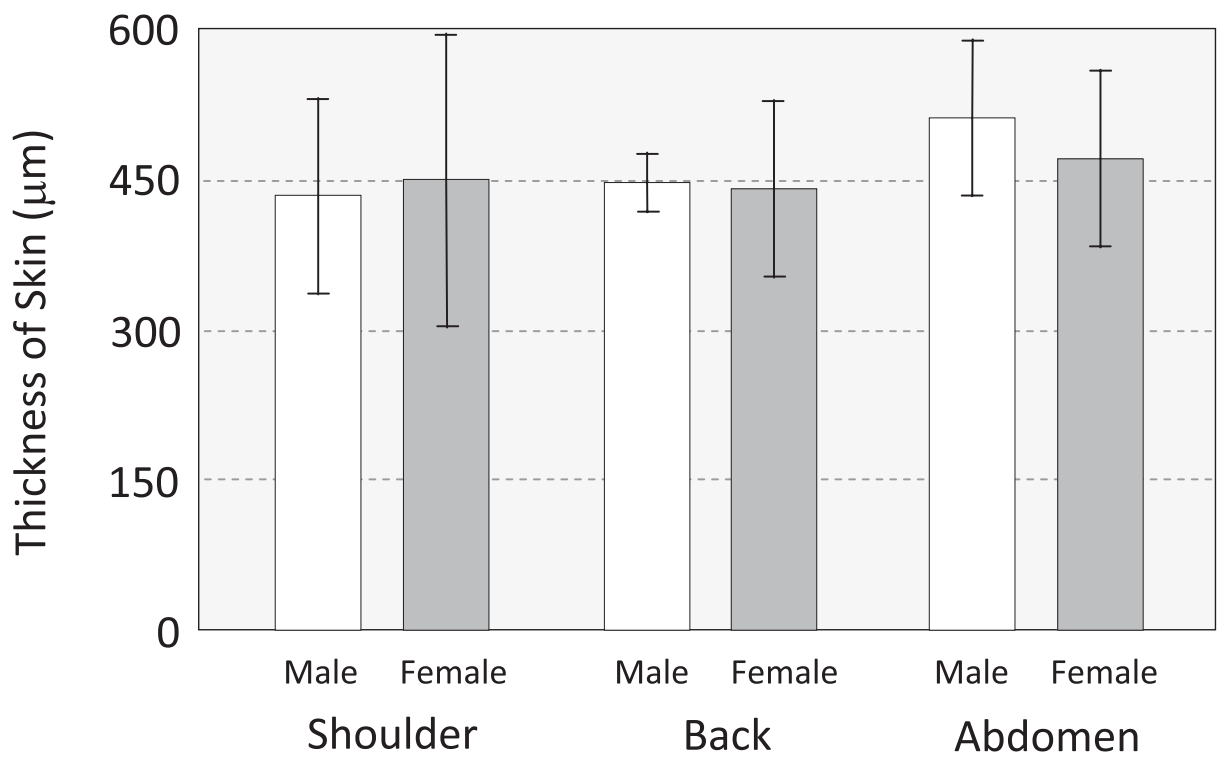

Fig. 2. Mean \pm SE of skin thickness for three body regions of male and female Hokkaido sika deer. 

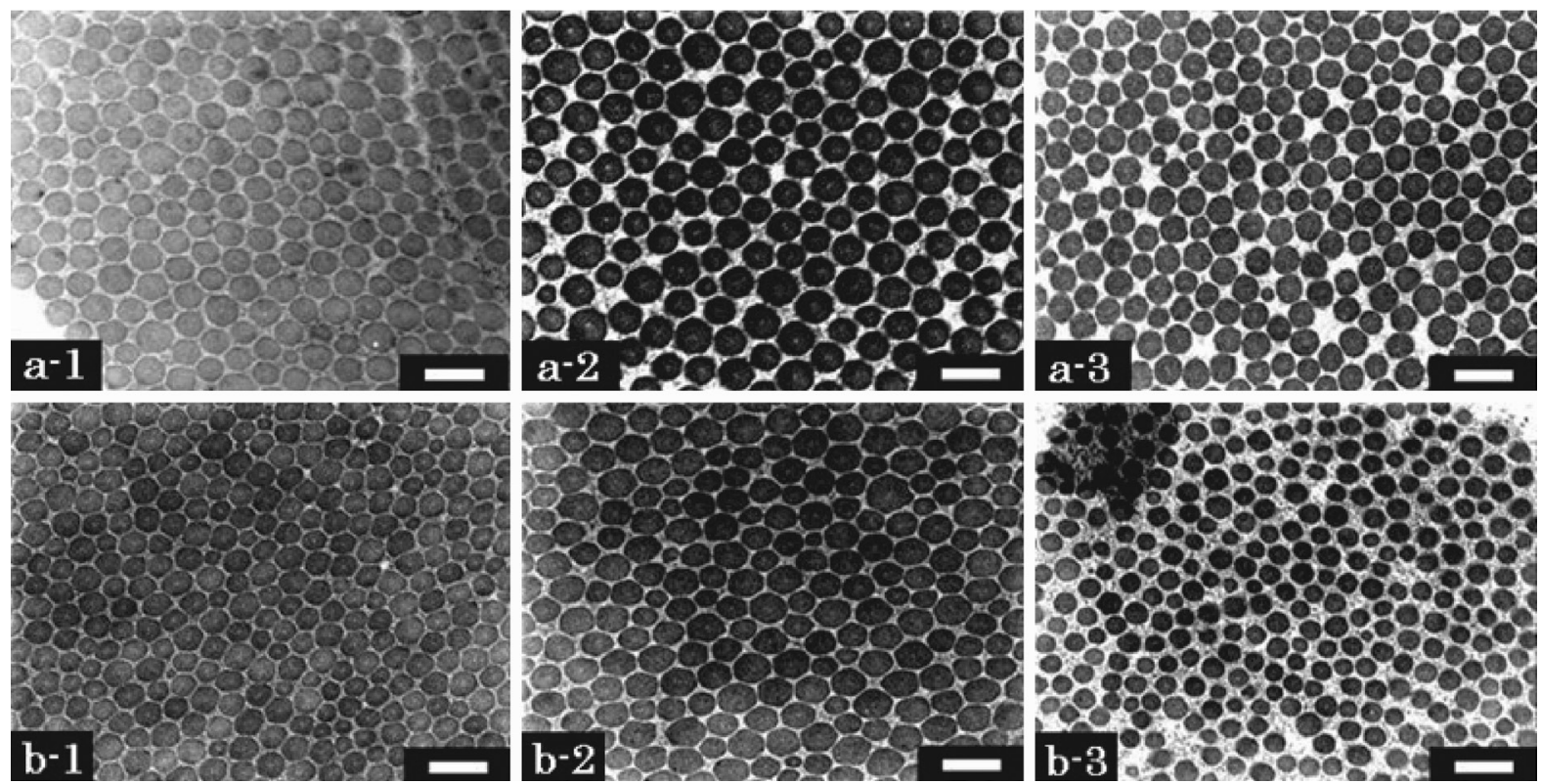

Fig. 3. Transmission electron micrographs showing collagen fibrils in Hokkaido sika deer skin. a: Male, b: Female, 1: Shoulder region, 2: Back region, and 3: Abdominal region, Bars indicate a scale of $200 \mathrm{~nm}$.

Table 1. Parameters of collagen fibrils in Hokkaido sika deer skin.

\begin{tabular}{|l|c|c|c|c|c|c|}
\hline \multirow{2}{*}{ Parameters } & \multicolumn{2}{|c|}{ Shoulder } & \multicolumn{2}{c|}{ Back } & \multicolumn{2}{c|}{ Abdomen } \\
\cline { 2 - 7 } & Male & Female & Male & Female & Male & Female \\
\hline $\begin{array}{l}\text { Fibril diameter } \\
(\mathrm{nm})\end{array}$ & $86.8 \pm 0.3$ & $81.4 \pm 0.4$ & $88.1 \pm 0.4$ & $87.5 \pm 0.6$ & $78.3 \pm 0.4$ & $72.3 \pm 0.5$ \\
\hline MAD (nm) & $90.2 \pm 1.7$ & $84.9 \pm 2.0$ & $92.1 \pm 2.0$ & $92.0 \pm 3.1$ & $81.4 \pm 1.9$ & $75.7 \pm 2.7$ \\
\hline CFI (\%) & $70.1 \pm 0.5$ & $71.8 \pm 1.0$ & $67.5 \pm 0.7$ & $71.1 \pm 1.2$ & $67.1 \pm 0.8$ & $64.3 \pm 1.5$ \\
\hline
\end{tabular}

Note: Data were shown as Mean \pm S.E.

TEM images are shown in Fig. 3. To avoid errors due to sectional obliquity, the minimal fibril diameter was always measured. The mean diameters of collagen fibrils, MADs, and CFIs are summarized in Table 1. Skin in the back region was thickest while that of the abdominal region was thinnest. Although the MAD of the abdominal skin was much lower than that of the other two regions within each sex, intra-sex comparisons between the same regions did not reveal any differences. It was apparent that the CFIs of the back and abdominal regions were lower than that of the shoulder region within either sex. However, the shoulder and back skin of male deer had lower CFIs than the respective skin of female deer.

Histograms representing the distributions of collagen fibril diameters measured from the electron micrographs in Fig. 3 are shown in Fig. 4. It can be seen that the distribution of shoulder skin of male deer has a broader peak (90 to $120 \mathrm{~nm}$ ) than that of female deer (70 to $100 \mathrm{~nm})$. In the back region, the distribution has only one peak at $100-110 \mathrm{~nm}$ in the male deer, and $90-100 \mathrm{~nm}$ in the female deer. The abdominal skin in both male and female deer has a peak at $70-80 \mathrm{~nm}$, but the amount of collagen fibrils with a diameter over $100 \mathrm{~nm}$ in the male deer was larger than in the female deer.

\section{Discussion}

The thickness of the outer skin layer of both male and female Hokkaido sika deer $(0.46 \pm 0.01 \mathrm{~mm})$ is not only 

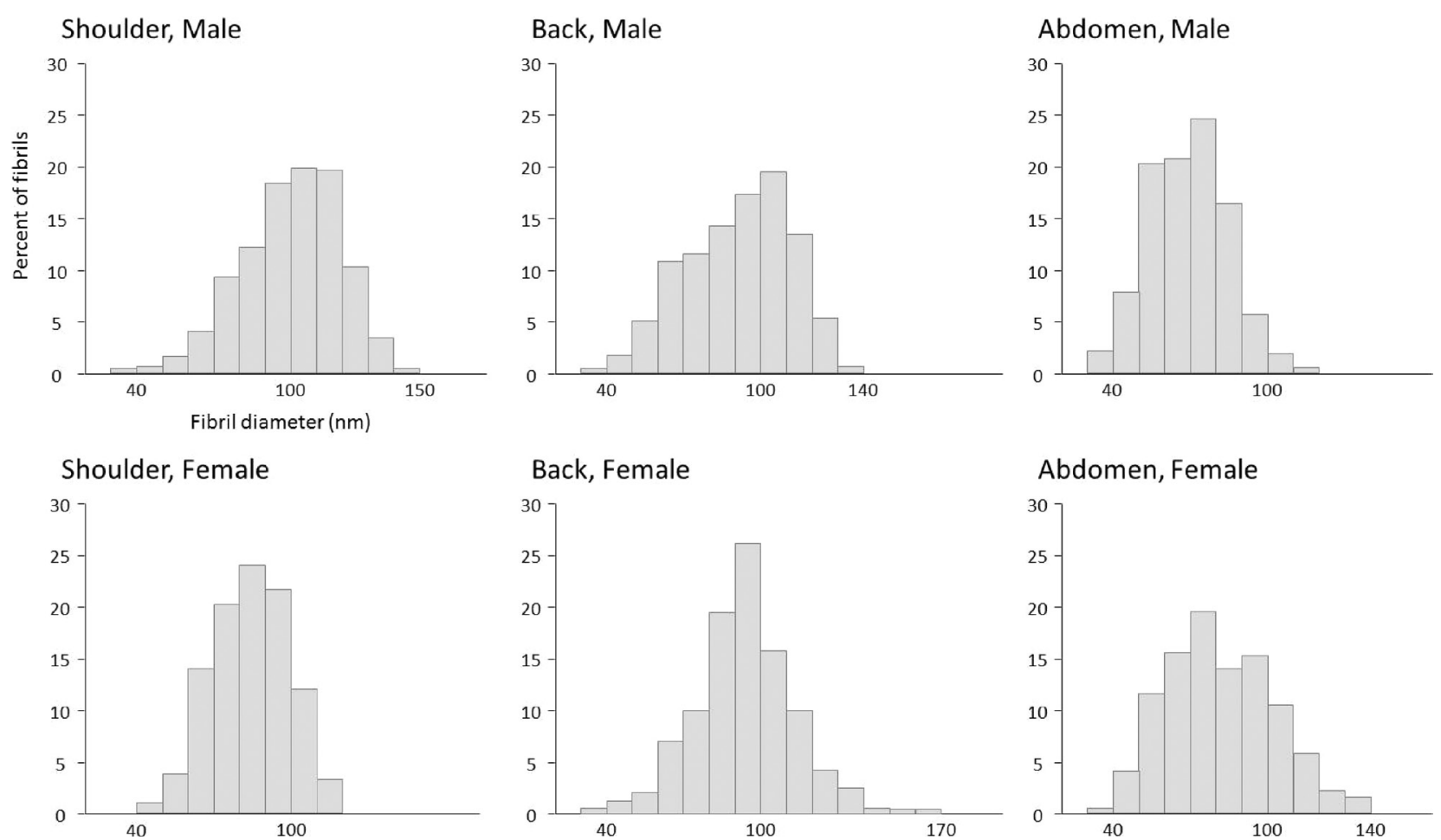

Fig. 4. Histograms showing collagen fibril diameter distribution in male and female Hokkaido sika deer skin.

uniform, but also comparatively thinner than that of pigs $(1-2 \mathrm{~mm})$ and cows $(5-7 \mathrm{~mm})$. For any region, flexibility of the skin generally requires collagen fibrils with a narrow diameter ${ }^{4}$. The average skin collagen fibril diameter for all regions of both sexes was approximately $82.4 \mathrm{~nm}$, while those of cow, pig, and horse skin were 112.6, 119.2, and $116.5 \mathrm{~nm}$, respectively. A higher number of smalldiameter collagen fibrils would probably contribute to increased flexibility, especially in the abdominal skin of the female deer.

Collagen fibrils are considered a basic unit of resistance of tensile strength, with the mechanical properties comprising fibril diameter and its distribution ${ }^{5}$. Thin fibrils provide elasticity through their ease of interaction within the extracellular matrix, and by suppressing movement among collagen fibrils. In contrast, thick fibrils provide strength through their many intermolecular cross-links ${ }^{6}$. Distributions of large-diameter collagen fibrils in the shoulder region of male deer highlight this region's dominance and importance versus that of female deer. Stronger shoulder skin would likely support male deer's aggressive, pugnacious behavior, especially in fights involving shoulder bumping. Of further note, female abdominal skin, with its broader distribution of small-diameter collagen fibrils, would have a higher flexibility for skin expansion, especially during pregnancy.
Although our data on the characteristic differences of the outer skin layer among these three body regions of male and female Hokkaido sika deer have the potential to support better utilization of deer skin for leather production, cross-regional studies on other specific histological properties, such as effective utilization of Hokkaido sika deer skin, are still necessary.

\section{Acknowledgements}

We would like to thank all the staff of the Nishiokoppe Wildlife Association, Hokkaido, Japan, for their assistance in the skin collection of our Hokkaido sika deer samples.

\section{References}

1) Flint MH, Craig AS, Reilly HC, Gillard GC, Parry DA. Collagen fibril diameters and glycosaminoglycan content of skins - indices of tissue maturity and function. Connect Tissue Res. 1984; 13(1):6981.

2) Yamamoto E, Hata D, Kobayashi A, Ueda H, Tangkawattana P, Oikawa M, Takehana K. Effect of beta-aminopropionitrile and hyaluronic acid on repair of collagenase-induced injury of the rabbit Achilles tendon. J Comp Path 2002; 126(2-3):161-70.

3) Sese M, Ueda H, Watanabe T, Yamamoto E, Hosaka Y, Tangkawattana P, Takehana K. Characteristics of collagen fibrils in the entire 
equine superficial digital flexor tendon. Okajimas Folia Anat Jpn 2007; 84(3):111-4.

4) Watanabe T, Imamura Y, Hosaka Y, Ueda H, Takehana K. Graded arrangement of collagen fibrils in the equine superficial digital flexor tendon. Connect Tissue Res 2007; 48(6):332-7.

5) Magnusson SP, Hansen P, Kjaer M. Tendon properties in relation to muscular activity and physical training. Scand J Med Sci Sports. 2003; 13(4):211-23.

6) Parry DA, Barnes GR, Craig AS. A comparison of the size distribution of collagen fibrils in connective tissues as a function of age and a possible relation between fibril size distribution and mechanical properties. Proc R Soc Lond B Biol Sci 1978; 203(1152):305-21. 
